SLAC-PUB-10687

hep-ph/0409042

September 2004

\title{
LITTLE OR NO HIGGS
}

\author{
T.G. RIZZO \\ Stanford Linear Accelerator Center, 2575 Sand Hill Rd. Menlo Park CA 94025, USA \\ E-mail: rizzo@slac.stanford.edu
}

Both Little Higgs and Higgsless Models provide new windows into the mysteries of electroweak symmetry breaking and lead to testable predictions at present and future colliders. Here we give a quick overview of three papers submitted to the ICHEP2004 meeting on these subjects.

\section{Monte Carlo Analysis of the Warped 5D Higgsless Model}

Higgsless models offer the possibility of electroweak symmetry breaking without the introduction of any explicit additional Higgs fields in the action within the extra dimensional framework. The various symmetries are broken via the application of appropriately chosen boundary conditions (BC) on the $5 \mathrm{D}$ gauge field wavefunctions. The role of the Goldstone bosons is played by the additional scalar component of the original 5D gauge fields which are 'eaten' to generate the massive 4D Kaluza-Klein(KK) tower of gauge fields. Unlike in orbifold models the would be zero mode can obtain a finite mass.

The most phenomenologically sucessful framework for a model of this type has been presented in Refs. ${ }^{1,2}$. In these models one takes the usual Rantall-Sundrum 5D warped setup $^{3}$ and places a Left-Right Symmetric $S U(2), \times S U(2)_{R} \times U(1)_{B-L}$ gauge theory in the bulk with the SM fermions being localized to the Planck brane. (The LR symmetry helps insure that $\rho$ parameter is very nearly unity.) The $\mathrm{BC}$ are chosen to break $S U(2)_{R} \times U(1)_{B-L} \rightarrow U(1)_{Y}$ on the Planck brane while simultaneously breaking $S U(2)_{L} \times S U(2)_{R} \rightarrow S U(2)_{D}$, the diagonal subgroup, on the $\mathrm{TeV}$ brane leaving just $U(1)_{Q}$. In addition, brane kinetic terms for the gauge fields corresponding to the unbroken generators on the two branes are also present: $S U(2)_{D}$ and $U(1)_{B-L}$ on the $\mathrm{TeV}$ brane and $S U(2)_{L}$ and $U(1)_{Y}$ on the Planck brane. The relative strength of these brane terms are described via a set of dimensionless parameters $\delta_{D, B, L, Y}$, which, together with $\kappa=g_{R} / g_{L}$, the ratio of the gauge couplings, are the free parameters of this model. Other model parameters can be traded for $M_{W, Z}$ and $G_{F}$ in various combinations.

There are a large number of constraints that such models must satisfy and there is a strong tension in this model between these various requirements: $(i)$ agreement with the precision electroweak data (which, since we are performing a tree level analysis, we interpret to mean the tree level SM), (ii) the bounds on the masses and couplings of KK excitations from direct and indirect(i.e., contact interaction) searches and (iii) perturbative unitarity $(\mathrm{PU})$ in $W_{L}^{+} W_{L}^{-}$elastic scattering. Recall that without the Higgs the SM violates perturbative unitarity at a scale of $\sqrt{s} \simeq 1.7 \mathrm{TeV}$; here the KK's must unitarize this scattering amplitude instead of the Higgs via $s$ - and $t$-channel exchanges. Naively this amplitude grows like $s^{2}$ but all such terms are cancelled due to gauge invariance when the various diagrams are combined thus leaving an $s$-like growth. The SM Higgs cancels these terms leaving an amplitude which has no power-like growth in $s$; here in this model the Higgs is absent so that this cancellation is non-trivial. In principle, the remaining amplitude that naively behaves as $s^{0}$ can still be large.

To see if an allowed model parameter space exists we ${ }^{2}$ generated via Monte Carlo techniques $\sim 5 \cdot 10^{6}$ sets of models de-

Work Supported by the Department of Energy Contract DE-AC02-76SF00515

Presented at the 32nd International Conference on High Energy Physics, 8/16/2004 - 8/22/2004, Beijing, China 


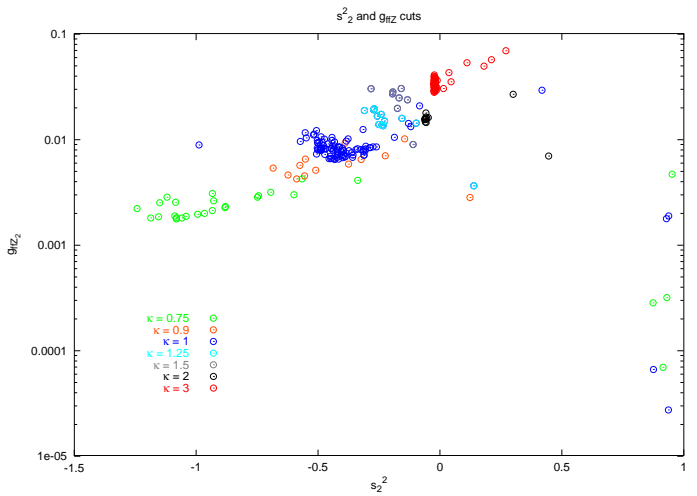

Figure 1. Mass and effective $\sin ^{2} \theta$ for the first KK excitation beyond the $Z$ for models passing the criteria discussed in the text.

scribed by the above parameters. We then asked whether all the constraints above could be satisfied simultaneously by passing them through a series of filters. We first demanded that all $g_{i}^{2}>0$ and that there be no ghost states in the spectrum. We then required the value of $\rho_{\text {eff }}$, defined via the $W$ and $Z$ couplings, to be within $0.5 \%$ of unity and that the various electroweak couplings be the same as their SM values at the same level of precision, $0.5 \%$. In our parameterization, deviations from the usual SM couplings are described by the $\rho_{\text {eff }}$ parameter and the existence of three different $\sin ^{2} \theta_{w}$ 's: the on-shell value $\sin ^{2} \theta_{O S}=1-M_{W}^{2} / M_{Z}^{2}$ using the observed $W$ and $Z$ masses as input, $\sin ^{2} \theta_{e g}=e^{2} / g_{W}^{2}$, where $g_{W}$ is defined through the $W$ coupling to the SM fermions with $e$ being the electric charge and $\sin ^{2} \theta_{\text {eff }}$ defined the the $Z$ pole couplings to fermions. We remind the reader that this is a tree level analysis and that these three quantities are a priori different in this model but are identical in the tree level SM. (They do differ in the SM at the loop level.) Next all TevII and LEPII constraints on the KK's must be satisfied including direct searches for KK resonances and the contact interaction effects induced via KK exchanges. Similarly, the couplings and masses of the first neutral KK must be such as to po- tentially lead to perturbative unitarity(PU). Certainly if the first neutral KK state is more massive than $\simeq 1.5 \mathrm{TeV}$ it will be too heavy to rescue unitarity. In addition, if the same KK couples mainly to hyperchage it will not couple to $W_{L}^{+} W_{L}^{-}$and thus will clearly fail in providing unitarity. Fig.1 shows that there are a reasonable set of models that survive these general requirements. (Note that tightening up the electroweak constraints, e.g., letting $0.5 \% \rightarrow 0.1 \%$, reduces the number of survivors by about a factor of 10.) Unfortunately, also requiring that there be no dangerous tachyons that couple strongly to SM fields removes almost all (but 6!) of these survivors. Tachyons of this dangerous type are found to exist when $\delta_{B, D}<0$ or when $\delta_{L}<<0$ which describes most of the survivors.

To examine $\mathrm{PU}$ for the remaining models we performed a straightforward evaluation of the $J=0$ partial wave amplitude, $a_{0}$, and required $\left|a_{0}\right|<1 / 2$ as usual. Unfortunately these models fail this requirement when $\sqrt{s} \geq 2 \mathrm{TeV}$ or so which is not much better than the SM with a Higgs. It is possible that this straightforward application of this now standardized test is too restrictive in the class of models under consideration and other tests may be more applicable. This is currently under active investigation.

\section{6D Higgsless Model in Flat Space}

It is interesting to examine the possible extensions of the 5D Higgsless model. Perhaps going to $6 \mathrm{D}$ may alleviate some of the problems of the 5D warped Higgsless model due to greater flexibility; the simplest $6 \mathrm{D}$ scenario, with only the SM in a flat space bulk, was considered in Ref!4 . Here the symmetries are broken via boundary conditions along the orthogonal additional directions in two steps, i.e., $S U(2)_{L} \rightarrow U(1)_{I_{3}}$, the third component of weak isospin, and $U(1)_{I_{3}} \times U(1)_{Y} \rightarrow$ 
$U(1)_{Q}$, as is shown in Fig.2. In addition to the two gauge couplings, $g, g^{\prime}$, and the radii of the two additional dimensions, $R_{i}$, there are two mass parameters in the $6 \mathrm{D}$ bulk action, $M_{L, Y}$, employed to produce fields and couplings with canonical 4D dimensionalities. Besides this bulk action, the conventional 4D SM gauge fields plus the SM fermion fields are localized at $(0,0)$ as boundary terms. In principle one imagines that $R_{1} \sim R_{2}$ and $M_{L} \sim M_{Y}$ so that no substantial parameter hierarchy exists. If the brane terms dominate those in the bulk then one has the inequality $g^{-2}, g^{\prime-2}>>\left(\pi M_{L, Y} R_{1,2}\right)^{2}$ which is the model limit examined in Ref! .

Note that the masses of the SM $W$ and $Z$ have somewhat different origins, i.e., whereas the $W$ mass arises from the BC's only on the $y_{1}=\pi R_{1}$ surface, the $Z$ mass also obtains contributions from the $y_{2}=\pi R_{2}$ surface. To leading order, e.g., one obtains $M_{W}^{2}=2 g^{2} M_{L}^{2} R_{2} / R_{1}$ whereas $M_{Z}^{2}=$ $2\left(g^{2}+g^{\prime 2}\right) M_{L}^{2} M_{Y}^{2}\left(R_{1} / R_{2}\right) /\left(M_{L}^{2}+M_{Y}^{2}\right)$. KK excitation masses go as $\sim 1 / R_{i}$, e.g., the first $W$ KK excitation has a mass of $1 / R_{1}$ to leading order. These mass relationships lead to a fine-tuning of the $\rho$ parameter since to leading order one obtains $\rho=(1+$ $\left.M_{L}^{2} / M_{Y}^{2}\right) R_{2}^{2} / R_{1}^{2}$ plus higher order terms that have been dropped. (To obtain this result the usual relation $\cos ^{2} \theta_{w}=g^{2} /\left(g^{2}+g^{\prime 2}\right)$ has been employed.) This ratio is naturally of order unity but is $\neq 1$ in general. In order to satisfy the electroweak constraints these set of parameters must thus be tuned to $\sim 1$ part in $10^{3}$. Once this tuning is made it can be shown that the other oblique parameters are suitably small.

In addition to this tuning problem there are a number of other unanswered questions about this model: $(i)$ The fermions remain massless as they are localized at $(0,0)$ where the SM remains unbroken. How are fermion masses to be generated? (ii) What are the couplings of the various $\mathrm{KK}$ states to the SM fermions? Are direct and indirect col-

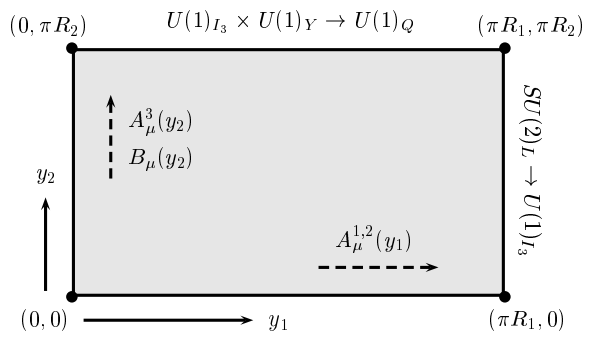

Figure 2. Symmetry breaking by boundary conditions of the $S U(2)_{L} \times U(1)_{Y} \mathrm{SM}$ in $6 \mathrm{D}$ flat space.

lider bounds on the KK spectrum easily satisfied? (iii) How are issues related to perturbative unitarity in $W_{L}^{+} W_{L}^{-}$elastic scattering addressed in this model? The answers to these and other questions may shed light on the phenomenological validity of this class of models.

\section{Fermion Completion of the Simplest Little Higgs Model}

In Little Higgs models the light SM-like Higgs is a psuedo-Goldstone boson which is protected from getting a mass by a pair of global symmetries. These same symmetries also remove the one-loop quadratic divegences in the Higgs sector that are present in the SM though these divergences remain at two-loop order. All such models have additional gauge, fermionic and scalar degrees of freedom at the $\mathrm{TeV}$ scale which play important roles in removing the quadratic divergences. In the simplest $S U(3)_{L} \times U(1)_{X}$ model of Kaplan and Schmalt 2 , the left-handed top quark becomes a member of an $S U(3)_{L}$ triplet representation, $3_{L}$, together with $b_{L}$ and an $S U(2)_{L}$ isosinglet quark with $Q=2 / 3, T_{L}$, of the form $(t, b, T)_{L}^{T}$; all $\mathrm{RH}$ fields are singlets as in the SM. Note that in addition to the $W, Z, \gamma$ of the SM there exists a new neutral hermitian gauge boson, as well as a pair of non-hermitian neutral gauge fields connecting $t_{L}$ and $T_{L}$ and a new pair of charged gauge fields connecting $b_{L}$ and $T_{L}$. Since the 
top quark yields the largest fermionic contribution to the Higgs quadratic divergence, here cancelled by the contribution of $T$, little attention is generally paid as to how the other SM fermions transform. This issue has been taken up by Kong 6 .

Kong has shown that there is a unique way to introduce the other SM fermions within the $S U(3) \times U(1)$ framework while maintaining anomaly freedom: ( $i$ ) The first two generations of LH-quarks are placed in anti-triplets, i.e., $\overline{3}_{L}$ 's, e.g., $(d, u, D)_{L}^{T}$, with their RH-partners remaining as singlets; here $D$ is an $S U(2)_{L}$ isosinglet $Q=-1 / 3$ state. (ii) All three generations of the LH-leptons are placed in triplets of the form $(\nu, \ell, N)_{L}^{T}$ while the RH partners of $\ell$ 's are singlets. Here $N$ is a neutral $S U(2)_{L}$ isosinglet state that can be of use in generating small neutrino masses. The SM-like Higgs fields lie in two anti-triplets and lead to interesting fermion mass patterns that can be generated by terms originating from dimension-4 and dimension5 operators.

Perhaps the most serious issue facing this scenario is the existence of FCNC's that arise after fermion mixing from a number of sources including the transformation of the new fermions into those of the SM via the emission of a $Z$. These occur due to the fact that the conventional Paschos-WeinbergGlashow conditions are no longer satisfied. Other FCNC sources also appear through the exchanges of the $S U(3)_{L}$ gauge bosons not present in the SM. Whether it is possible to satisfy the existing experimental constraints while simultaneously satisfying quartic divergence cancellation, precision electroweak data and collider bounds remains unknown. This model is in need of further study.

\section{Conclusions}

While it is clear that our understanding of electroweak symmetry breaking is incomplete there is a growing number of new ideas com- ing on the market. Soon, data from the LHC and the ILC will help elucidate this situation. Hopefully by the time of ICHEP2008 we will already have a much clearer view of the physics behind electroweak symmetry breaking.

\section{Acknowledgments}

Work supported in part by the Department of Energy, Contract DE-AC02-76SF00515.

\section{References}

1. C. Csaki, C. Grojean, L. Pilo and J. Terning, Phys. Rev. Lett. 92, 101802 (2004) [arXiv hep-ph/0308038; G. Cacciapaglia, C. Csaki, C. Grojean and J. Terning, arXiv hep-ph/0401160 C. Csaki, C. Grojean, J. Hubisz, Y. Shirman and J. Terning, Phys. Rev. D 70, 015012 (2004) [arXiv hep-ph/0310355.

2. For details of the analysis and original references, see J. L. Hewett, B. Lillie and T. G. Rizzo, arXiv hep-ph/0407059. H. Davoudiasl, J. L. Hewett, B. Lillie and

T. G. Rizzo, JHEP 0405, 015 (2004); H. Davoudiasl, J. L. Hewett, B. Lillie and T. G. Rizzo, Phys. Rev. D 70, 015006 (2004) [arXiv hep-ph/0312193.

3. L. Randall and R. Sundrum, Phys. Rev. Lett. 83, 3370 (1999) [arXiv hep-ph/9905221.

4. For details, see S. Gabriel, S. Nandi and G. Seidl, arXiv hep-ph/0406020

5. D. E. Kaplan and M. Schmaltz, JHEP 0310,

(2003) [arXiv hep-ph/0302049; see also M. Schmaltz, arXiv hep-ph/0407143

6. For a full analysis, see O. C. W. Kong, arXiv hep-ph/0312060,

arXiv hep-ph/0308148 and arXiv hep-ph/0307250. 